\title{
Dynamics analysis of pilot's upper limb manipulation process
}

\author{
Xiufang YANG ${ }^{*},{ }^{* *}$, Youchao SUN*, Daxu ZHAO**, Zidong YANG ${ }^{* *}$, Lijian YAO ${ }^{* *}$ and Yundong GUO* \\ * College of Civil Aviation, Nanjing University of Aeronautics and Astronautics, Nanjing 211106, China \\ E-mail: syz_nju@163.com \\ ** School of Engineering, Zhejiang A \& F University, Lin'an 311300, China \\ E-mail: daxu@nuaa.edu.cn
}

Received: 12 December 2017; Revised: 8 June 2018; Accepted: 3 October 2018

\begin{abstract}
A 6-degree-of-freedom (DOF) model of a pilot's upper limb is established in this study. A kinematics analysis is performed by using the screw theory and the product of exponential formula. Kane's equation in screw form, which is a concise form with a definite physical meaning, is used to analyze the dynamic characteristics of a pilot's upper limb. In the Mathematica environment, the man-machine system consisting of a pilot and a joystick is taken as the analysis object to simulate the joystick pushing and pulling processes of a pilot at the 50th percentile of Chinese body dimensions. The analysis yields the angular velocity and angular acceleration curves of the joint, which indicate that the manipulation comfort is rather good. The actual posture data during the pilot manipulation process are measured. Through a comparison with the output data, the correctness of the simulation analysis is verified. The torque curve reflects that the torque of the shoulder joint is greater than that of the elbow joint, and the changing tendency conforms to the actual motion law. Therefore, the correctness of Kane's equation in screw form is verified. At the same time, the results can serve as a theoretical basis for evaluating a pilot's manipulation comfort and as an important reference for cockpit layout design.
\end{abstract}

Keywords : Screw theory, Product of exponential formula, Kane's equation, Mathematica, Joystick

\section{Introduction}

With the increased complexity and integration of modern cockpits, the pilot work load has also increased. Apart from manipulating the throttle lever, joystick, pedal, etc., a pilot also needs to manipulate, e.g., switches and buttons. Although an autopilot system can reduce the workload of a pilot, the pilot still plays a dominant role in the event of an emergency. Therefore, accurate pilot manipulation can guarantee flight safety (Xue et al., 2013).

Relevant statistics presented by the FAA (1990) show that more than two-thirds of aviation accidents are caused by the anthropogenic factors of the pilot. Researchers believe that the attribution of human errors entirely to pilots is incorrect because an unreasonable cockpit layout design can also lead to pilot mistakes (Foyle and Hooey, 2008). For example, pilots can manipulate an aircraft by pushing and pulling a joystick. During the push-pull process, a pilot's arm applies force to the joystick, which, in turn, has a counterforce on the shoulder, elbow, and wrist. If the joystick is improperly arranged, the pilot may be subjected to a rather large counterforce for a long time, causing fatigue and reducing pilot reaction speed and accuracy (Cao and Yang, 2010), thereby affecting the flight safety of the aircraft and undermining the pilot's physical and mental health (Kong, 2017). Therefore, a kinetic analysis of the pilot manipulation process must be carried out.

Studies concerning manipulation process analysis and manipulation posture comfort evaluation include the following: Jung et al (1995), in line with the human reach requirements and considering the redundancy of human joint degrees of freedom, assumed the upper limb to be a multipoint articulated chain and established a comfort assessment model based on upper limb size. Wang et al (2008) established a rigid body model of pilot upper limbs by using the dimensions of the Chinese male pilot population and Chinese manhood anthropometric data. They used SimMechanics for the dynamics simulation of the pilot push-pull manipulation process to obtain the torque of the upper limb joints 
and determined the three-dimensional comfortable manipulation area of the upper limbs according to comfort criteria. Ma et al (2008) established the pilot comfort evaluation model based on the Jack software static posture library and studied the force and torque output of the upper limbs during manipulation via an artificially produced manipulating conversion force. Marler et al. (2009) conducted an overall multiobjective optimization of the virtual human posture from the perspective of the human body joint posture, musculoskeletal discomfort conditions and human physical exertion. Considering that poor working posture can result in operators suffering from musculoskeletal disorders (MSDs), Ma et al (2009) conducted a multiobjective optimization of work posture from the perspective of workers' physical exertion in practical work and fatigue and recovery. Yang et al (2012) established a complete 5-degree-of-freedom (DOF) human upper limb operation comfort model. They proposed a modeling method for the RULA-based human upper limb comfortable manipulation optimization model and obtained the comfort distribution curve for collective manipulation. Jung et al (2010) and Lee et al (2013) measured the human body data of nearly a hundred Korean helicopter pilots and studied the pilots' operational comfort zones at the 5th, 50th and 95th percentiles. Wang et al (2013) simulated the action of lever operation using the Anybody software and obtained data concerning the shoulder joint force, elbow joint force and force acting on the hand for a joystick under different circumstances. They then compared the data within the group and arrived at the conclusion that when one, two, or even all three variables changed, the values of these three forces, among which the shoulder joint force was the maximum one and varied most significantly, were negatively correlated with the position factor. By using Kane's dynamic equation, Deng et al (2017) established the upper limb dynamics model to determine the joint torque, and using NASA's power model, they introduced the joint torque into the joint comfort assessment, determining the overall comfort assessment of upper limbs with different joint angle combinations.

The above studies utilized anthropometric data and the static reach and comfort area for cockpit human-machine interface design and displayed the control equipment arrangement to make flights safer and more comfortable. However, aircraft manipulation is dynamic. For example, manipulatable parts within a cockpit are located within a pilot's static reach, which indicates only that they are manipulatable and does not show whether it is convenient for a pilot to operate them or whether the movements needed to do so are smooth and comfortable. The dynamic characters of the manipulation, i.e., the size of the manipulation torque, motion track, manipulation speed and acceleration, must still be studied. Therefore, a 6-DOF model of a pilot's upper limbs is established in this study to perform, using the screw theory and product of exponential formula, dynamics analysis of a pilot's upper limbs. Kane's equation in screw form, which is concise and has a definite physical meaning, is used to analyze the dynamics characteristics. In the Mathematica environment, taking the man-machine system consisting of a pilot and a manipulator as the analysis object, we simulate and analyze a pilot's manipulation of a joystick to determine the angular velocity, angular acceleration and driving torque of the joint, which provides a basis for evaluating a pilot's manipulation comfort.

\section{Pilot's upper limb model}

Human upper limbs can be divided into the upper arm, forearm, and hand, which are connected via the shoulder, elbow, and wrist joints. The upper arm, forearm and hand can be simplified as a rigid body. Ignoring the freedom of the fingers, upper limbs have 6 DOFs, as shown in Fig. 1. 


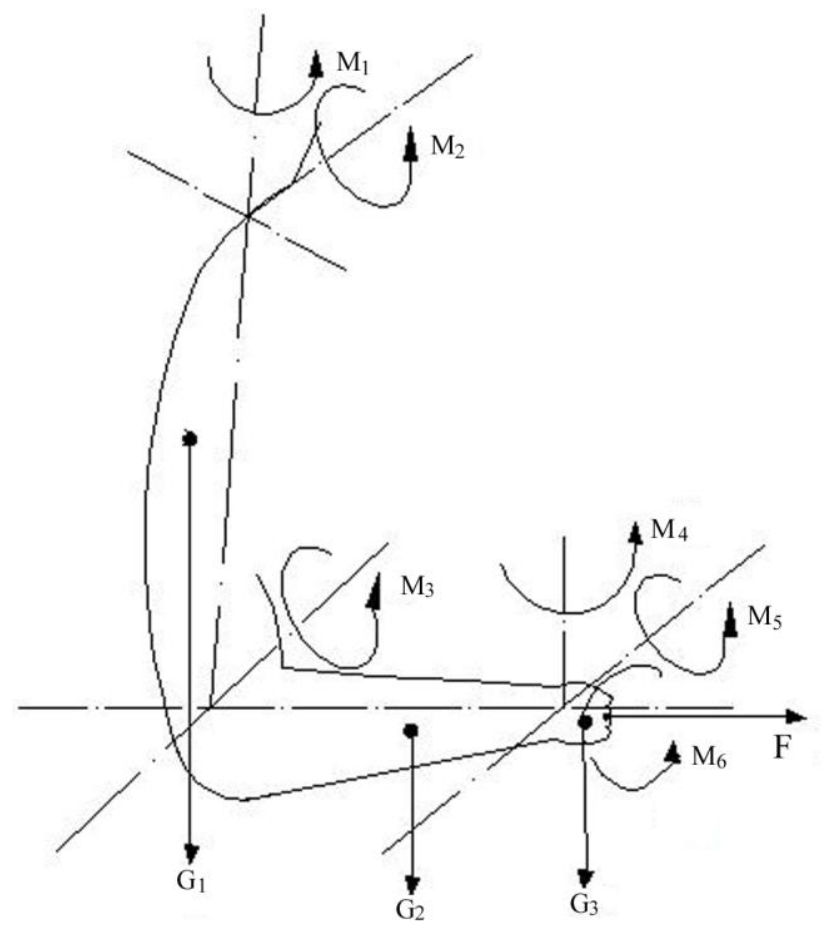

Fig 1 Upper limb model. $M_{i}$ represents the joint torque, $G_{i}$ denotes the gravity, and $F$ represents the manipulation force

\section{Kinematics model based on screw theory}

\subsection{Rigid body motion based on screw theory}

The product of exponential formula of screw theory, which was first introduced into robot modeling by Brockett (1984), is used to derive the kinematics equation. The method has the following advantages: 1) It can describe the motion of a rigid body as a whole in the inertial coordinate system and provide a complete, obvious geometric description, avoiding the singularity caused by describing the local coordinate system via the D-H parameter method. 2) It can be used to better determine the conditions for generating multiple solutions and the number of multiple solutions and to better define its clear geometric significance when the product of exponential formula of screw theory is used for the inverse solution. Therefore, the screw method clearly describes the geometric meaning of rigid body motion, avoiding the drawbacks of mathematical symbol abstraction and greatly simplifying the process of analyzing the mechanisms and the solution process (Chen et al., 2014; Rocha et al., 2017). It is therefore widely used in the kinematics and dynamics analysis of new robots or mechanisms (Selig, 2008; Sariyildiz et al., 2011).

All potential postures that a single rigid body possesses in space constitute a special orthogonal group $S O(3)$, and the group elements are a rotation transformation matrix $\boldsymbol{R}$ around the origin of coordinates. The semidirect product of $S O$ (3) and vector space $\boldsymbol{P}$ constitutes a Lie group $S E(3)$. The group element $\mathbf{K}$ denotes all possible configurations of the rigid body, the homogeneous matrix of which is expressed as $K=\left[\begin{array}{cc}R & P \\ 0 & 1\end{array}\right] \in R^{4 \times 4}$. SE(3) in the Lie group is a smooth manifold $\mathcal{M}$, and the smooth path corresponds to a parametric transformation sequence of different relative positions of the rigid body. The $S E(3)$ element in the Lie group takes a derivative with respect to time to obtain the algebra se(3) of the Lie group. Element $\hat{\xi}=\left[\begin{array}{cc}\tilde{\omega} & v \\ 0 & 0\end{array}\right]$ in $\operatorname{se}(3)$ is referred to as the motion screw, where 
$\tilde{\omega}=\left[\begin{array}{ccc}0 & -\omega_{3} & \omega_{2} \\ \omega_{3} & 0 & -\omega_{1} \\ -\omega_{2} & \omega_{1} & 0\end{array}\right] \in R^{3 \times 3}$ represents translational motion. For the sake of computational convenience, the 6-dimensional vector form $\xi=\left[\begin{array}{ll}\boldsymbol{\omega} & \mathbf{v}\end{array}\right]^{\mathrm{T}} \in \mathrm{R}^{6}$ in $s e(3)$ is generally used as the spin representation of the velocity change of the rigid body (Zhao et al., 2016). The motion screw $\xi$ reflects the relative motion between two adjacent rigid bodies in the chained system, corresponding to the motion screw when all other joints are fixed at the zero position in addition to the joint in question (Richard et al., 1998):

$$
\xi_{i}=\left\{\begin{array}{cc}
{\left[\begin{array}{cc}
-\omega_{i} \times q_{i} & \omega_{i}
\end{array}\right]^{T}} & \text { rotational jo int } \\
{\left[\begin{array}{ll}
v_{i} & 0
\end{array}\right]^{T}} & \text { prismatic jo int }
\end{array}\right.
$$

\subsection{Kinematics analysis of upper limbs}

Because the pilot's chest and abdomen are fixed to the seat, the basic coordinate system is $0-\mathrm{XYZ}$. The origin of coordinates is the reference position of the seat's neutral position. The $\mathrm{X}$ forward direction points from the back to the front chest, the $\mathrm{Y}$ forward direction, from the trunk axis to the outside, and the $\mathrm{Z}$ forward direction, from the bottom to the top. The tool coordinate system is o-xyz, and the origin of coordinates is the wrist. The xyz direction is consistent with the basic coordinate system. The arm stretched out horizontally is the initial state, $\mathrm{k}_{\mathrm{st}}(0)=\left[\begin{array}{cc}I_{3} & P(0) \\ 0 & 1\end{array}\right]$, where $\mathrm{P}(0)=\left[\begin{array}{cccc}1 & 0 & 0 & 0 \\ 0 & 1 & 0 & l_{1}+l_{2} \\ 0 & 0 & 1 & l_{0} \\ 0 & 0 & 0 & 1\end{array}\right]$. The screw motion coordinates of each joint are $\omega_{1}=\omega_{4}=\left[\begin{array}{lll}0 & 0 & 1\end{array}\right]^{T}$, $\omega_{2}=\omega_{3}=\omega_{5}=\left[\begin{array}{lll}-1 & 0 & 0\end{array}\right]^{T}$, and $\varpi_{6}=\left[\begin{array}{lll}0 & 1 & 0\end{array}\right]^{T}$. The points of each joint axis are as follows: $q_{1}=\left[\begin{array}{lll}0 & 0 & l_{0}\end{array}\right]^{T} \quad, \quad q_{2}=\left[\begin{array}{lll}0 & 0 & l_{0}\end{array}\right]^{T} \quad, \quad q_{3}=\left[\begin{array}{lll}0 & l_{1} & l_{0}\end{array}\right]^{T} \quad, \quad q_{4}=\left[\begin{array}{lll}0 & l_{1}+l_{2} & l_{0}\end{array}\right]^{T}$, $q_{5}=\left[\begin{array}{lll}0 & l_{1}+l_{2} & l_{0}\end{array}\right]^{T}$, and $q_{6}=\left[\begin{array}{lll}0 & l_{1}+l_{2} & l_{0}\end{array}\right]^{T}$. One motion screw $\zeta_{\mathrm{i}}$ is constructed for each joint. The shoulder, elbow and wrist are regarded as rotational joints. For rotational joints, the equation $\zeta_{\mathrm{i}}=\left[\begin{array}{c}-\varpi_{i} \times q_{i} \\ \varpi_{i}\end{array}\right]$ is used. The motion screw $\zeta_{\mathrm{i}}$ and $e^{\xi_{i} \theta_{i}}$ of each joint are as indicated in Table 1.

Table 1. Motion screw $\zeta_{\mathrm{i}}$ and $e^{\xi_{i} \theta_{i}}$

\begin{tabular}{llllll}
\hline$i$ & \multicolumn{1}{c}{$\zeta_{\mathrm{i}}$} & $e^{\xi_{i} \theta_{i}}$ \\
\hline 1 & {$\left[\begin{array}{lllllllll}0 & 0 & 0 & 0 & 0 & 1\end{array}\right]^{T}$} & {$\left[\begin{array}{cccc}\cos \theta_{1} & -\sin \theta_{1} & 0 & 0 \\
\sin \theta_{1} & \cos \theta_{1} & 0 & 0 \\
0 & 0 & 1 & 0 \\
0 & 0 & 0 & 1\end{array}\right]$} \\
\hline
\end{tabular}




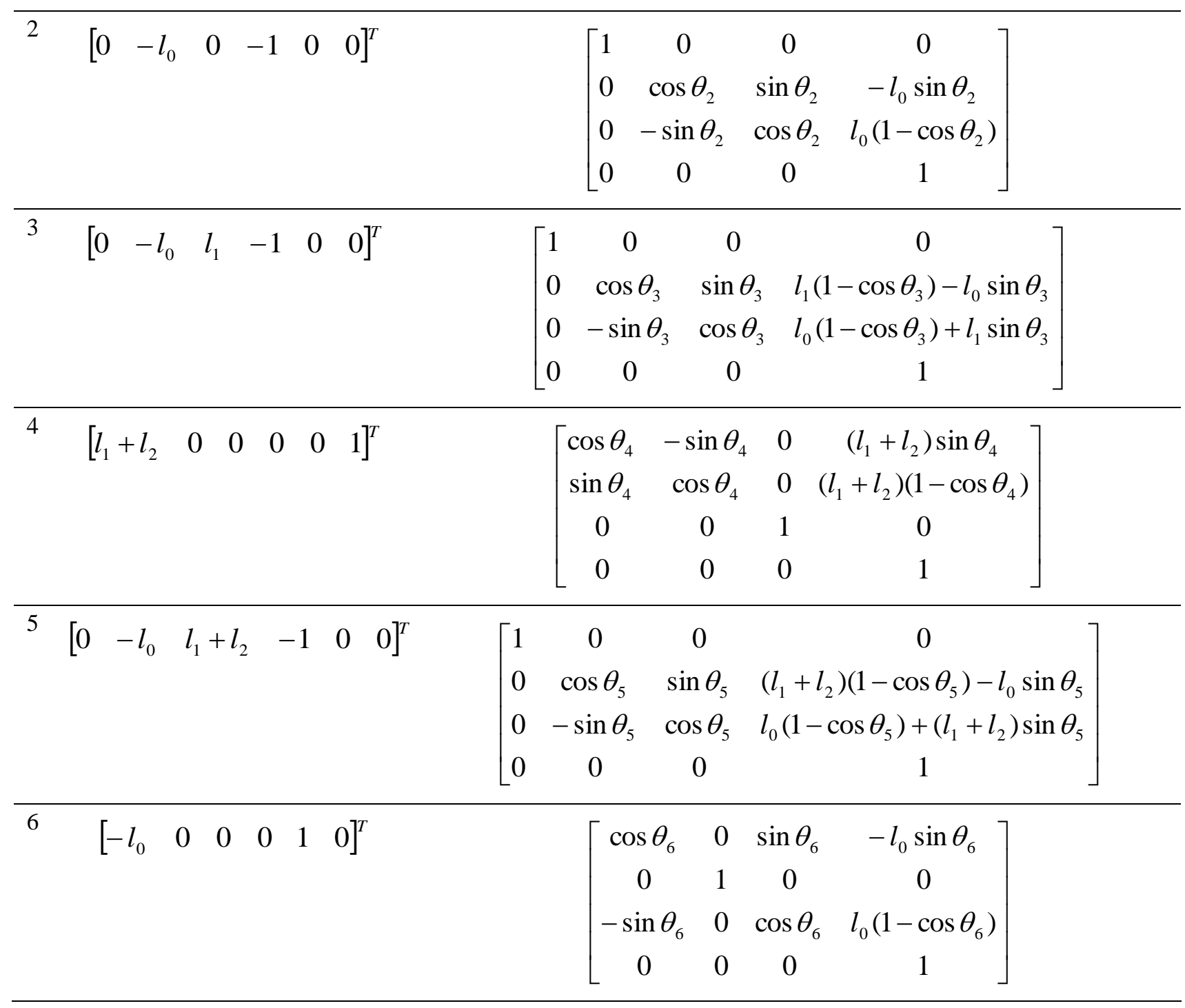

Rigid body motion is relayed from the basic coordinate system $\mathrm{S}$ to $\mathrm{T}$ via a series of body-fixed coordinate systems. The configuration of the rigid body in $\mathrm{S}$ is obtained via successive transformations of the exponential product of all low-order bodies:

$$
k_{s t}(\theta)=e^{\hat{\xi_{1}} \theta_{1}} e^{\hat{\xi_{2}} \theta_{2}} \cdots e^{\hat{\xi}_{n} \theta_{n}} k_{s t}(0)
$$

According to Eq. 2, the kinematic mapping of the exponential product is derived by superimposing the joint motions:

$$
k_{s t}(\theta)=e^{\hat{\xi_{1}} \theta_{1}} e^{\hat{\xi_{2}} \theta_{2}} \cdots e^{\hat{\xi}_{6} \theta_{6}} k_{s t}(0)=\left[\begin{array}{cc}
R(\theta) & P(\theta) \\
0 & 1
\end{array}\right]
$$

where $R(\theta)$ indicates the terminal attitude, which is a three-factorial square matrix represented as follows: $\mathrm{c}_{\mathrm{i}}=\cos \theta_{\mathrm{i}}$, $\mathrm{s}=\sin \theta_{\mathrm{i}}, \mathrm{c}_{\mathrm{ij}}=\cos \left(\theta_{\mathrm{i}}+\theta_{\mathrm{j}}\right)$, and $\mathrm{s}_{\mathrm{ij}}=\sin \left(\theta_{\mathrm{i}}+\theta_{\mathrm{j}}\right)$. Element $\mathrm{r}_{\mathrm{ij}}$ of $R(\theta)$ is as follows:

$\mathrm{r}_{11}=\mathrm{c}_{6}\left(\mathrm{c}_{1} \mathrm{c}_{4}-\mathrm{s}_{1} \mathrm{c}_{23} \mathrm{~s}_{4}\right)+\mathrm{s}_{6}\left(\mathrm{~s}_{1} \mathrm{~s}_{23} \mathrm{c}_{5}+\mathrm{s}_{1} \mathrm{c}_{23} \mathrm{c}_{4} \mathrm{~s}_{5}+\mathrm{c}_{1} \mathrm{~s}_{4} \mathrm{~s}_{5}\right)$,

$\mathrm{r}_{12}=-\mathrm{c}_{5}\left(\mathrm{~s}_{1} \mathrm{c}_{23} \mathrm{c}_{4}+\mathrm{c}_{1} \mathrm{~s}_{4}\right)+\mathrm{s}_{1} \mathrm{~s}_{23} \mathrm{~s}_{5}$,

$\mathrm{r}_{13}=\mathrm{c}_{6}\left[-\mathrm{c}_{5} \mathrm{~s}_{1} \mathrm{~s}_{23}-\left(\mathrm{c}_{23} \mathrm{c}_{4} \mathrm{~s}_{1}+\mathrm{c}_{1} \mathrm{~s}_{4}\right) \mathrm{s}_{5}\right]+\left(\mathrm{c}_{1} \mathrm{c}_{4}-\mathrm{c}_{23} \mathrm{~s}_{1} \mathrm{~s}_{4}\right) \mathrm{s}_{6}$,

$\mathrm{r}_{21}=\mathrm{c}_{6}\left(\mathrm{c}_{4} \mathrm{~s}_{1}-\mathrm{c}_{1} \mathrm{c}_{23} \mathrm{~s}_{4}\right)-\left[\mathrm{c}_{1} \mathrm{c}_{5} \mathrm{~s}_{23}+\left(\mathrm{c}_{1} \mathrm{c}_{23} \mathrm{c}_{4}-\mathrm{s}_{1} \mathrm{~s}_{4}\right) \mathrm{s}_{5}\right] \mathrm{s}_{6}$,

$\mathrm{r}_{22}=\mathrm{c}_{5}\left(\mathrm{c}_{1} \mathrm{c}_{23} \mathrm{c}_{4}-\mathrm{s}_{1} \mathrm{~s}_{4}\right)-\mathrm{c}_{1} \mathrm{~s}_{23} \mathrm{~s}_{5}, \mathrm{r}_{23}=\mathrm{c}_{6}\left[\mathrm{c}_{1} \mathrm{c}_{5} \mathrm{~s}_{23}+\left(\mathrm{c}_{1} \mathrm{c}_{23} \mathrm{c}_{4}-\mathrm{s}_{1} \mathrm{~s}_{4}\right) \mathrm{s}_{5}\right]+\left(\mathrm{c}_{4} \mathrm{~s}_{1}+\mathrm{c}_{1} \mathrm{c}_{23} \mathrm{~s}_{4}\right) \mathrm{s}_{6}$,

$\mathrm{r}_{31}=-\left(\mathrm{c}_{6} \mathrm{~s}_{23} \mathrm{~s}_{4}\right)-\left(\mathrm{c}_{23} \mathrm{c}_{5}-\mathrm{c}_{4} \mathrm{~S}_{23} \mathrm{~s}_{5}\right) \mathrm{s}_{6}, \mathrm{r}_{32}=-\left(\mathrm{c}_{4} \mathrm{c}_{5} \mathrm{~s}_{23}\right)-\mathrm{c}_{23} \mathrm{~s}_{5}, \mathrm{r}_{33}=\mathrm{c}_{6}\left(\mathrm{c}_{23} \mathrm{c}_{5}-\mathrm{c}_{4} \mathrm{~S}_{23} \mathrm{~s}_{4}\right)-\mathrm{s}_{23} \mathrm{~s}_{4} \mathrm{~s}_{6}$. 
$\mathrm{P}(\theta)$ is the terminal position, with $p(\theta)=\left[\begin{array}{c}-\sin \theta_{1} *\left(l_{1} * \cos \theta_{2}+l_{2} * \cos \left(\theta_{2}+\theta_{3}\right)\right) \\ \cos \theta_{1} *\left(l_{1} * \cos \theta_{2}+l_{2} * \cos \left(\theta_{2}+\theta_{3}\right)\right) \\ l_{0}-l_{1} * \sin \theta_{2}-l_{2} * \sin \left(\theta_{2}+\theta_{3}\right)\end{array}\right]$.

\subsection{Jacobian and velocity transformation}

For upper limbs with three joints and $6 \mathrm{DOFs}, k_{s t}(\theta)$ indicates the configuration space of the terminal hand. The space instantaneous velocity can be expressed as follows:

$$
V_{S i}^{S}=\dot{k_{S i}} k_{S i}^{-1}=\left[\begin{array}{c}
\omega_{S i}^{S} \\
v_{S i}^{S}
\end{array}\right]
$$

The adjoint transformation matrix and dual transformation matrix forms of the two adjacent bodies $A_{i}$ and $A_{i-1}$ are as follows:

$$
\left\{\begin{array}{c}
A d_{k_{i, i-1}}(\xi)=\left[\begin{array}{cc}
R_{i, i-1} & 0 \\
-\tilde{p} R_{i, i-1} & R_{i, i-1}
\end{array}\right]\left[\begin{array}{l}
\omega \\
v
\end{array}\right] \\
A d_{k_{i, i-1}^{*}}^{*}\left(\xi^{*}\right)=\left[\begin{array}{cc}
R_{i, i-1}^{-1} & R_{i, i-1}^{T} \tilde{p}^{T} \\
0 & R_{i, i-1}^{-1}
\end{array}\right]\left[\begin{array}{l}
\tau \\
f
\end{array}\right]
\end{array}\right.
$$

$V_{S i}^{S}$ can be converted to the body coordinate system $i$ using the dual and adjoint transformation operator $A d_{k}^{*}$ :

$$
V_{S i}^{i}=A d_{k_{S i}}^{*} V_{S i}^{S}=\dot{k_{S i}} k_{S i}^{-1}=\left[\begin{array}{c}
\omega_{S i}^{i} \\
v_{S i}^{i}
\end{array}\right]
$$

A linear relation exists between the terminal hand velocity and the velocity of each joint, and the instantaneous velocity screw can be derived via linear superposition of the instantaneous velocity of each joint:

$$
V_{S T}^{T}=\dot{k_{S T}} k_{S T}^{-1}=\left[\begin{array}{c}
\omega_{S T}^{S} \\
v_{S T}^{S}
\end{array}\right]=\sum_{i=1}^{6}\left(\frac{\partial k_{S T}}{\partial \theta_{i}} k_{S T}^{-1}\right) \cdot \dot{\theta}_{i}
$$

The mapping relation between the terminal velocity $V_{S T}^{T}$ and joint velocity $\theta$ is $V_{S T}^{T}==J_{S T}^{S} \cdot \dot{\theta}_{i}$, where $J_{S T}^{S}$ is the space Jacobian matrix of the screw form. Therefore,

$$
J_{S T}^{S}=\left[\frac{\partial k_{S T}}{\partial \theta_{1}} k_{S T}^{-1} \cdots \frac{\partial k_{S T}}{\partial \theta_{6}} k_{S T}^{-1}\right]=\left[\xi_{1}^{S} \cdots \xi_{6}^{S}\right]
$$

According to Eq. 6 and the kinematic positive solution, the vector expression of the space Jacobian matrix is obtained:

$$
\xi_{k i}^{s}=\frac{\partial k_{S i}}{\partial_{\theta_{i}}} k_{S i}^{-1}=e^{\xi_{1} \theta_{1}} \cdots e^{\xi_{i-1} \theta_{i-1}}\left(\xi_{i}\right) k_{S i}(0) k_{S i}^{-1}=e^{\xi_{1} \theta_{1}} \cdots e^{\xi_{i-1} \theta_{i-1}}\left(\xi_{i}\right)=A d_{\left(e^{\xi \theta \theta_{1}} \ldots e^{\xi_{i-1} \theta_{i-1}}\right)} \xi_{i}
$$

According to Eq. 6, the velocity screw of the ith joint can be converted to the body-fixed coordinate system via the dual adjoint transformation operator. The body Jacobian of $\mathrm{A}_{i}$ in the system can be derived after the conversion:

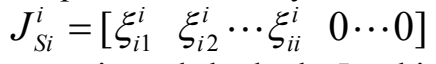

Both the space Jacobian matrix and the body Jacobian matrix are functions of time $t$, depending on the current configuration of the overall system.

\section{Kinetic equation of the screw form}

Kane's equation of the rigid body system is (Li et al., 2014): 


$$
F_{j}+F_{j}^{*}=0 \quad j=1,2, \cdots n
$$

where n denotes the DOFs, $F_{j}$ is the generalized active force, and $F_{j}^{*}$ is the generalized inertial force. The following equations can be derived:

$$
\left\{\begin{array}{l}
F_{j}=\sum_{i=1}^{n} f_{i} v_{i}^{(j)} \\
F_{j}^{*}=\sum_{i=1}^{n} f_{i}^{*} v_{i}^{(j)}
\end{array}\right.
$$

where $f_{i}$ is the active force screw, $f_{i}^{*}$ is the inertial force screw, and $v_{i}^{(j)}$ is the jth partial velocity screw relative to the inertial system. The key to solving Kane's equation is the partial velocity screw. $\theta=\left[\dot{\theta}_{1} \cdots \dot{\theta}_{6}\right]^{T}$ is selected as the generalized velocity of the system, and $J_{S i}^{i}$, the body Jacobian matrix of $\mathrm{A}_{i}$, is selected as the partial velocity screw. Each column of $\xi_{i j}^{i}$ indicates the partial velocity screw of the corresponding joint. The partial velocity is incorporated into Eq. 12 to derive:

$$
\left\{\begin{array}{c}
F_{j}=\sum_{i=1}^{n} f_{i} \xi_{i j}^{i} \\
F_{j}^{*}=\sum_{i=1}^{n} f_{i}^{*} \xi_{i j}^{i}
\end{array}\right.
$$

Mathematica can be used to calculate the relevant parameters of the above algorithm. Due to limited space, only the Jacobian matrices of the shoulder joint and elbow joint are provided below:

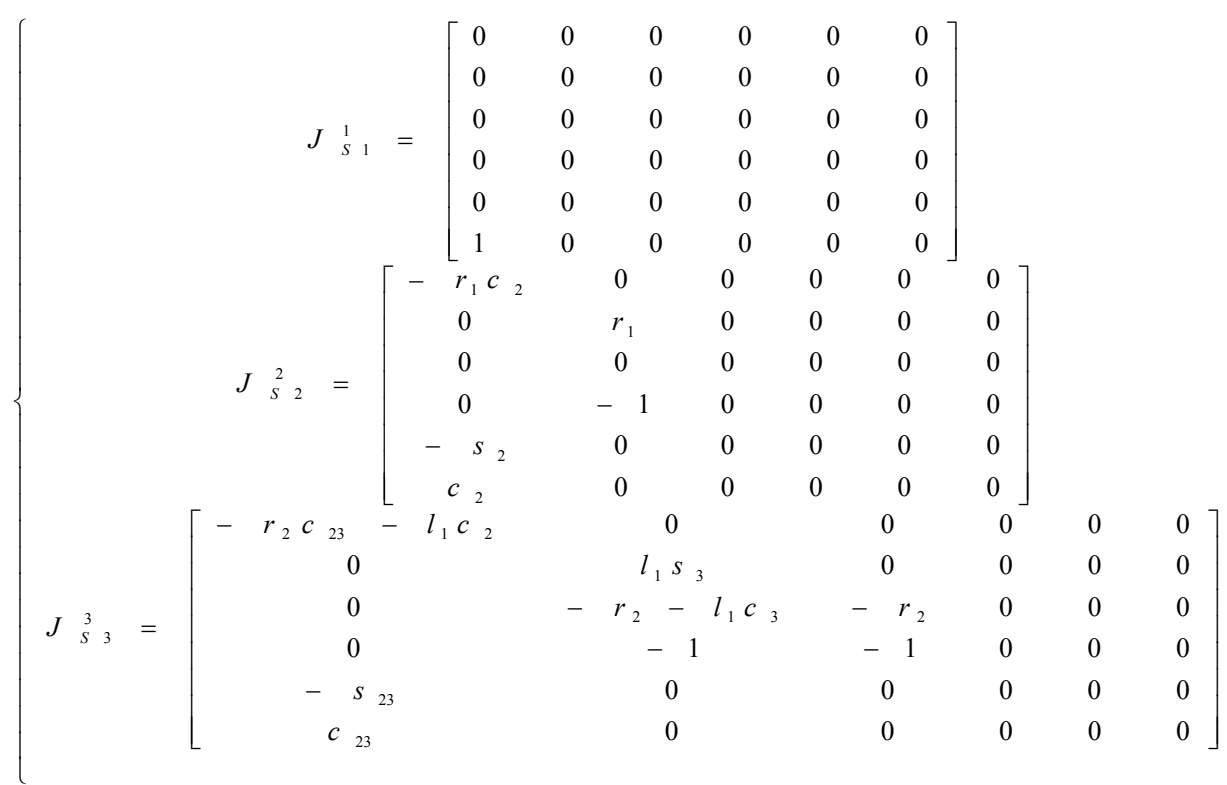

The active force screw acting on the centroid can be determined via the upper limb structure. The shoulder and elbow joint equation is as shown in Eq. 15, where $\tau_{i}$ represents the driving torque of each joint:

$$
\left.\begin{array}{c}
f_{1}=\left[0,0,-m_{1} g,-\tau_{2}, 0, \tau_{1}\right]^{T} \\
f_{2}=\left[0, m_{2} g s_{2},-m_{2} g c_{2}, \tau_{3}-\tau_{2}, 0,0\right]^{T} \\
f_{3}=\left[0, m_{3} g s_{23},-m_{3} g c_{23},-\tau_{3},-\tau_{4}, 0\right]^{T}
\end{array}\right\}
$$

The rigid body centroid acceleration and angular acceleration can be obtained based on the centroid acceleration of the rigid body and the angular acceleration of the connecting rod. Then, the inertia force screw of each rigid body can be derived. The active force screw and inertia force screw are incorporated into Eq. 13 to derive the driving torque for each joint.

\section{Kinetic simulation analysis of upper limb manipulation and real measurement}




\subsection{Kinematics simulation analysis}

According to the literature (Lu et al., 2013), the fiftieth percentile of human body dimensions is selected in this study. The parameters of each part of the upper limbs are shown in Table 2 (GJB 4856-2003, 2013).

Table 2. Dimensions of each part of a pilot's body (unit: $\mathrm{mm}$ )

\begin{tabular}{cc}
\hline Item & 50\% of the Chinese pilot population \\
\hline Sitting shoulder height $\left(l_{0}\right)$ & 0.608 \\
Upper arm $\left(l_{1}\right)$ & 0.315 \\
Forearm $\left(l_{2}\right)$ & 0.235 \\
Hand $\left(l_{3}\right)$ & 0.184 \\
\hline
\end{tabular}

The biomechanical parameters of the upper limbs are shown in Table 3 (Lu et al., 2013; Chen et al., 2016).

Table 3. Biomechanical parameters of each upper limb part

\begin{tabular}{ccccccc}
\hline Name & Mass $/ \mathrm{kg}$ & Length $(\mathrm{m})$ & Centroid position $(\mathrm{m})$ & \multicolumn{3}{c}{ Rotational inertia $\left(\mathrm{kg} \cdot \mathrm{mm}^{2}\right)$} \\
\cline { 5 - 7 } & & & & $\mathrm{X}$ & $\mathrm{Y}$ & $\mathrm{Z}$ \\
\hline Upper arm & 1.539 & 0.315 & 0.147 & 12370.87 & 12805.82 & 1600.2 \\
Forearm & 0.766 & 0.235 & 0.104 & 3227.9 & 3114.7 & 785.06 \\
Hand & 0.377 & 0.184 & 0.036 & & & \\
\hline
\end{tabular}

According to the literature (Lu et al., 2013; Wang et al., 2013), during upper limb manipulation of the pilot, the joint torque is largest when pushing and pulling the joystick. This study carries out a kinematic analysis of a pilot pushing and pulling a joystick. In the process of pulling the joystick, the fingers and palm are assumed to be stationary relative to the joystick. Thus, the movement of the joystick can be regarded as the movement of the hand. No relative motion for the hand and forearm exists. Meanwhile, a rotation exists between the upper arm and forearm, and a small-range rotation exists for the shoulder joint (Wang et al., 2013). Simulation of the upper arm while pulling the joystick is conducted using Mathematica. The joystick size and range of motion come from the simulation cockpit size (Fig. 2). The top coordinate of the joystick is $(40,22,23)$, and the joystick has a width of $4 \mathrm{~cm}$, a length of $6 \mathrm{~cm}$, and a height of $20 \mathrm{~cm}$.

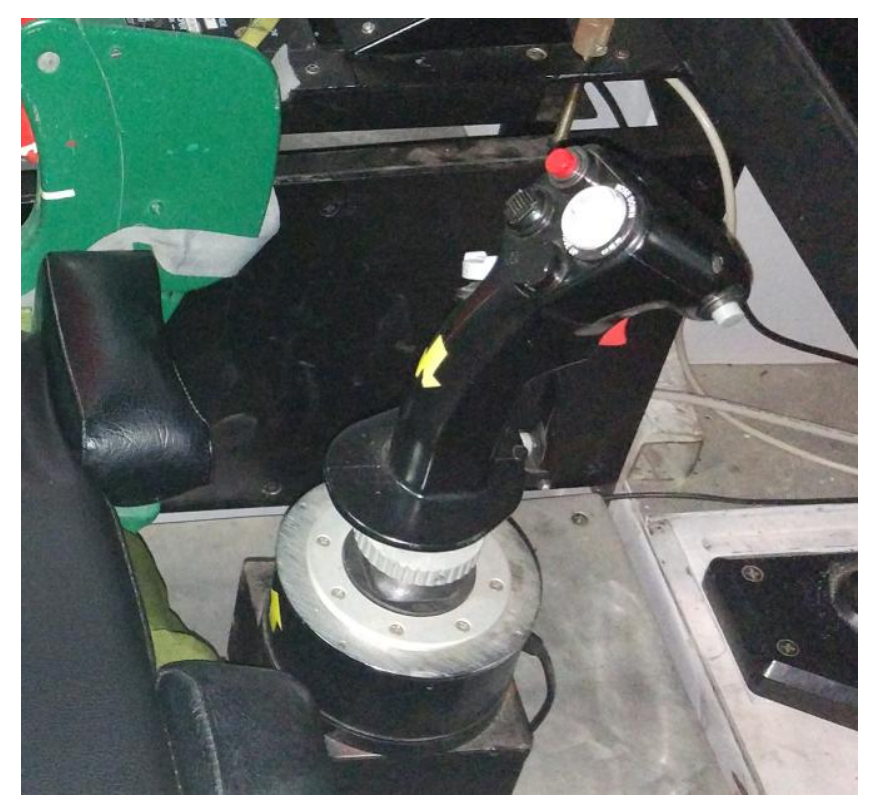

Fig. 2 Aircraft cockpit joystick

The manipulation is commenced after $10 \mathrm{~s}$ of simulation. The angular velocity curves of the shoulder's two DOFs (in the $\mathrm{X}$ and $\mathrm{Z}$ directions) are as shown in Figure 3. The velocity in the $\mathrm{X}$ direction is shown to decrease, and that in the $\mathrm{Z}$ direction first increases and then decreases. The angular velocity curve of the elbow's one DOF (in the $\mathrm{X}$ 
direction) is as shown in Fig. 4, in which the velocity decreases followed by a reverse increase.

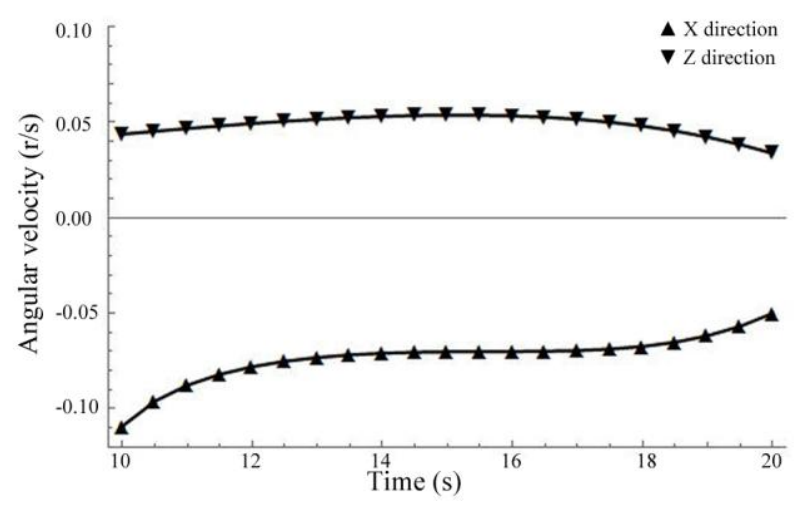

Fig. 3 Angular velocity curve of shoulder joint

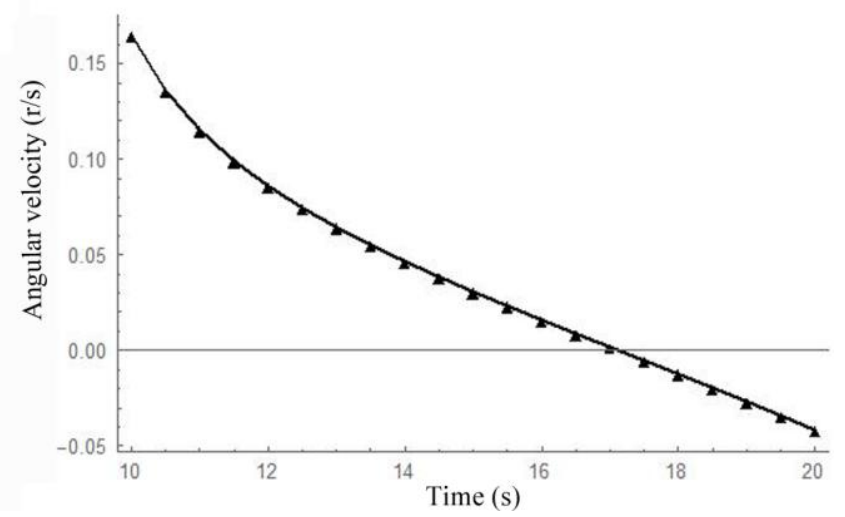

Fig. 4 Angular velocity curve of elbow joint

Using the obtained velocity, the angular acceleration is determined by deriving the time in the basic coordinate system. The angular acceleration of the shoulder joint is shown in Fig. 5, in which the acceleration in the $\mathrm{X}$ direction first decreases and then increases, while that in the $\mathrm{Z}$ direction gradually decreases and then increases. Figure 6 shows that the acceleration of the elbow joint gradually increases in the X direction and finally approaches a slope of zero. The smoother acceleration curve indicates smoother, easier manipulation and greater pilot comfort (Liu, 2016). Therefore, the figure clearly shows that in the process of pulling the joystick, no sudden change in acceleration occurs; thus, the manipulation comfort is rather good.

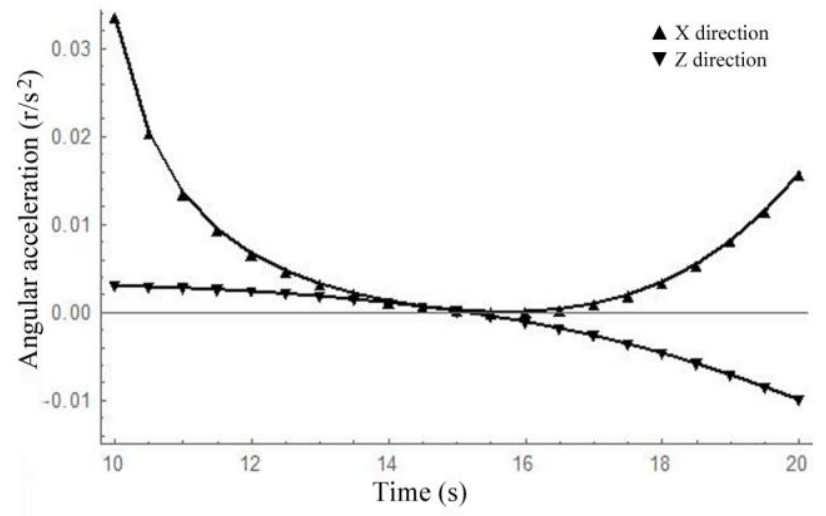


Fig. 5 Angular acceleration curve of shoulder joint

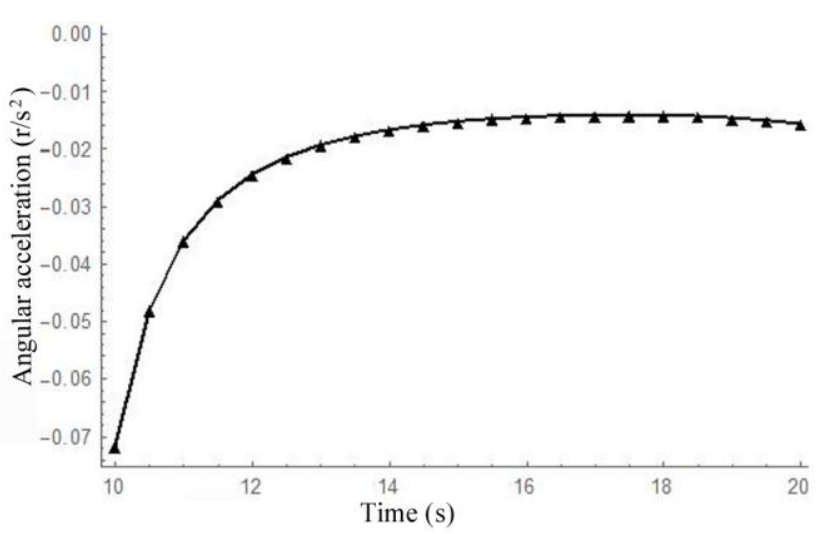

Fig. 6 Angular acceleration curve of elbow joint

\subsection{Measurement of the posture data during the manipulation process}

The accuracy of the simulation analysis is confirmed by comparing the motion position data output during the manipulation process and the simulation data through obtaining the movement during the manipulation process by means of a human posture capture system.

\subsubsection{Experimental setup}

The experimental setup includes one simulated cockpit device, 1 control signal acquisition system, and 1 inertial action tracking and measuring system. The simulated cockpit device is based on a certain type of military aircraft and can simulate flight events such as take-off, climbing, landing and other key tasks. The simulated cockpit can also be used for research on cockpit ergonomics design. The simulated cockpit platform is shown in Fig. 7. The area marked by the red line is the simulated cockpit console and the control signal acquisition system.

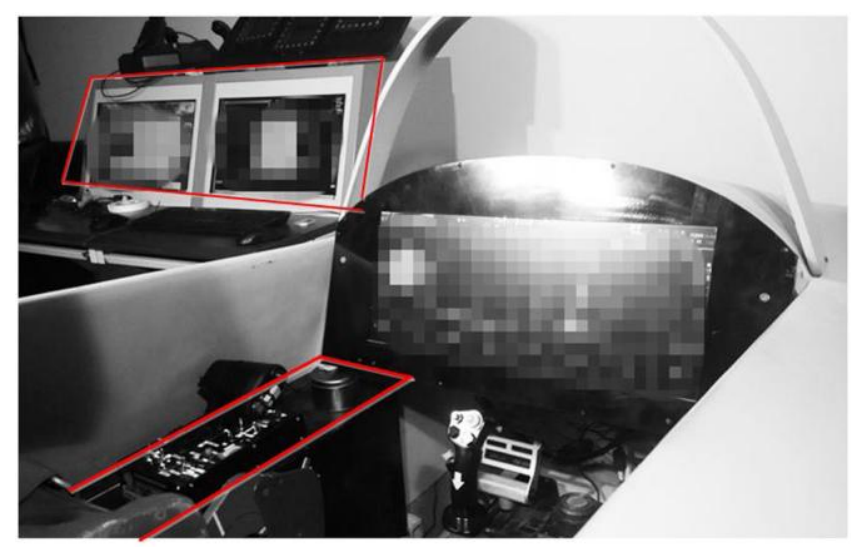

Fig. 7 Simulated cockpit platform

Fig. 8 shows the inertia action tracking and measuring device, which realizes real-time, high-precision and all-posture measurements. 


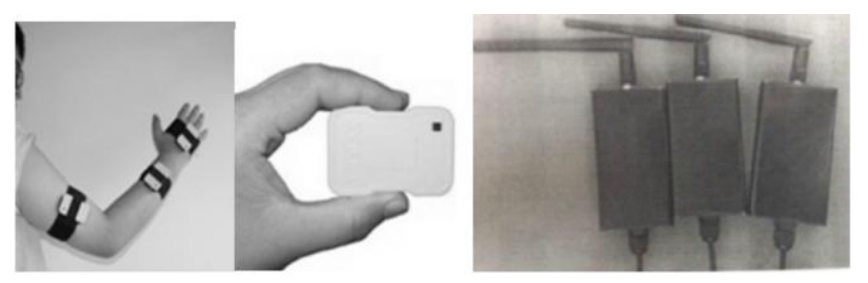

Fig. 8 Inertia action tracking device and signal receiver

\subsubsection{Experiment procedure}

The experimenters are intern pilots $(\mathrm{n}=10)$ who have received good training on a ground flight simulator. The dimensions of the pilots are close to 50th percentile, and the pilots are right handed with normal visual or corrected visual acuity. The flight route is selected as the Nanjing Lukou International Airport, and the whole flight process includes three stages, namely, take-off, cruising and approach.

The three receivers are connected with a USB wiring connector, which is in turn connected to the computer. The receiver drive is installed. The correspondence between the sensor and installation position is in accordance with the standard wearing style and is shown in Fig. 9. The MocapW.exe software is run on the server first, and then, settings are selected in the MocapW.exe interface (first, the experimenter selects "Settings" to open the panel on the menu, selects "Wireless" for the bus type in the lower left corner, and clicks "Connect" for connection). After making the connection, the experimenter performs a "Tpose" posture and clicks "Quick Calibrate" on the server panel (the rest is not required to be performed). If the experimenter needs to record, the "Save Log" button is pressed, as shown in Fig. 10. MotionBox.exe is run to display the 3D human body model.

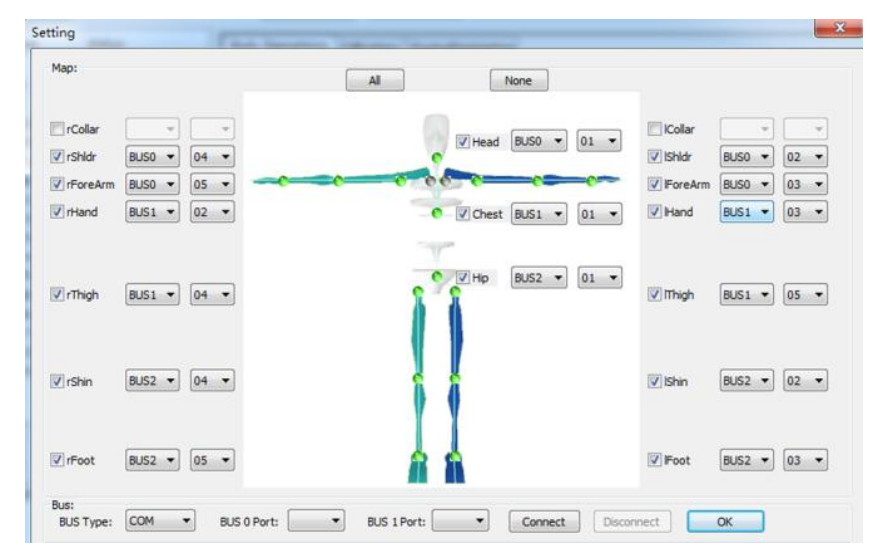

Fig. 9 Server-side bus setting interface 


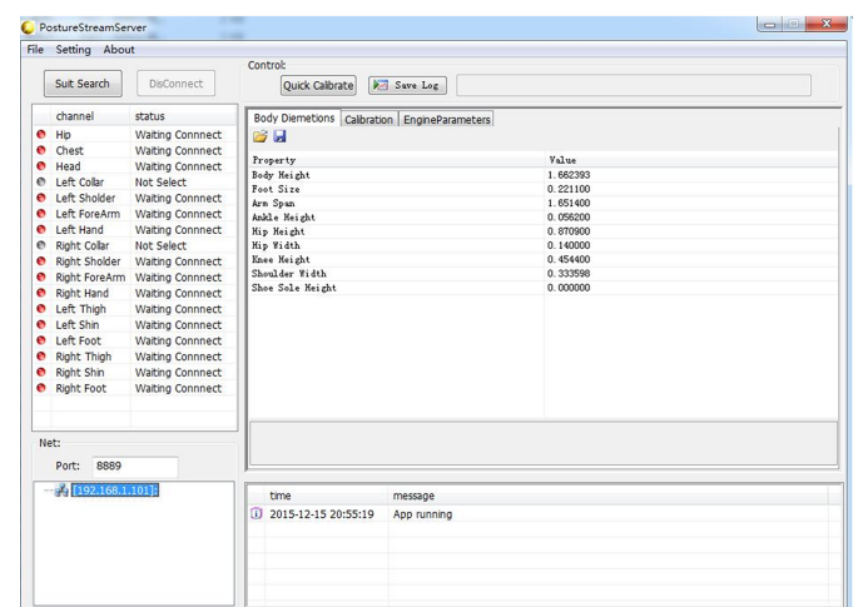

Fig. 10 Posture stream server panel

The '.bin' document recorded by MocapW. exe is changed to 'test1.Bin' and copied to the same level folder of jiexi.exe to generate 'test1.Txt'. The format of 'test1.Txt' is as shown in Fig. 11. The times indicate the sampling times of the sensor, and ser00(X), ser00(Y), and ser00(Z) are the real-time positions of sensor 0 .

$\begin{array}{lllll}\text { Times } & \text { Time } & \text { ser00(X) } & \text { ser00(Y) } & \text { ser00 (Z) } \\ 0000: & 20: 54: 41.691 & 0.000000 & -0.000000 & 0.000000 \\ 0001: & 20: 54: 41.738 & 0.000000 & -0.000000 & 0.000000 \\ 0002: & 20: 54: 41.785 & 0.000000 & -0.000000 & 0.000000 \\ 0003: & 20: 54: 41.831 & 0.000000 & -0.000000 & 0.000000\end{array}$

Fig. 11 Test1.txt format

\subsubsection{Data analysis}

The action of pulling the joystick is measured by the inertia action tracking device to output the movement position data of the upper arm, forearm, and hand (the data are provided in Supplementary File 1). The program in Mathematica is used to simulate the action of the upper arm pulling the joystick to output the position data. A 10 20s time interval is selected for comparison. The results are as shown in Fig. 12-14.

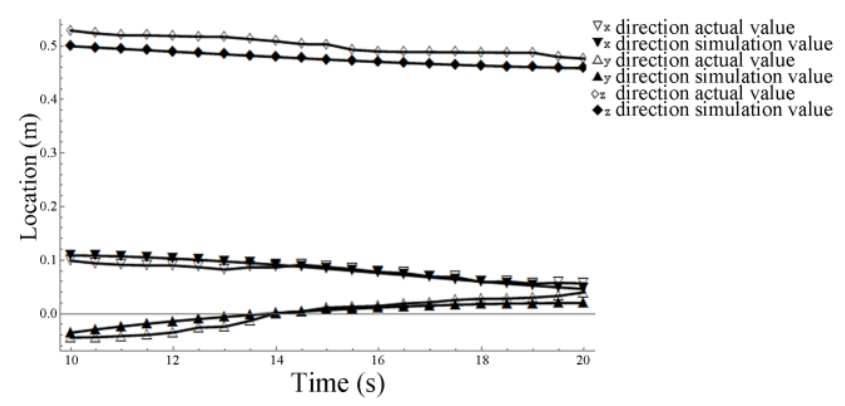

Fig. 12 Upper arm movement data and simulation analysis results

Fig. 12 shows that during the joystick pulling process, the coordinate values of the upper arm decrease gradually along the $\mathrm{X}$ direction, reversely decrease along the $\mathrm{Y}$ direction first and then increases, and gradually decrease along 
the $\mathrm{Z}$ direction with negligible variation. The actual values are basically consistent with the simulation values, with the same change tendency.

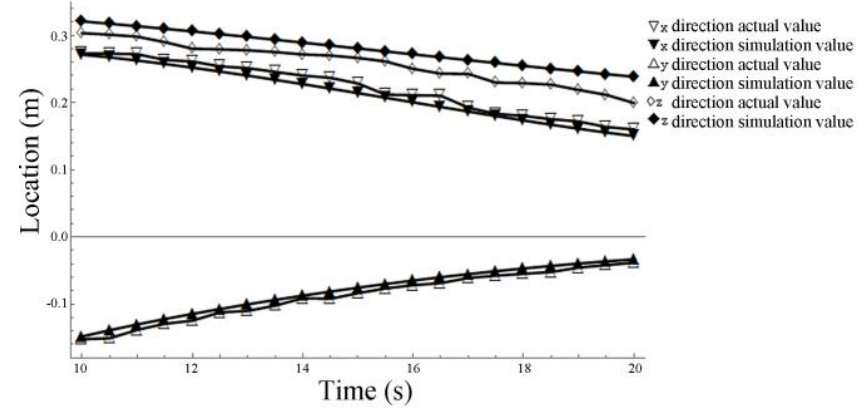

Fig. 13 Forearm movement position data and simulation analysis results

Fig. 13 shows that during the joystick pulling process, the coordinate values of the forearm decrease along the $\mathrm{X}$ direction, reversely decrease along the $\mathrm{Y}$ direction, and decrease along the $\mathrm{Z}$ direction. The actual values are basically consistent with the simulation values with the same change tendency.

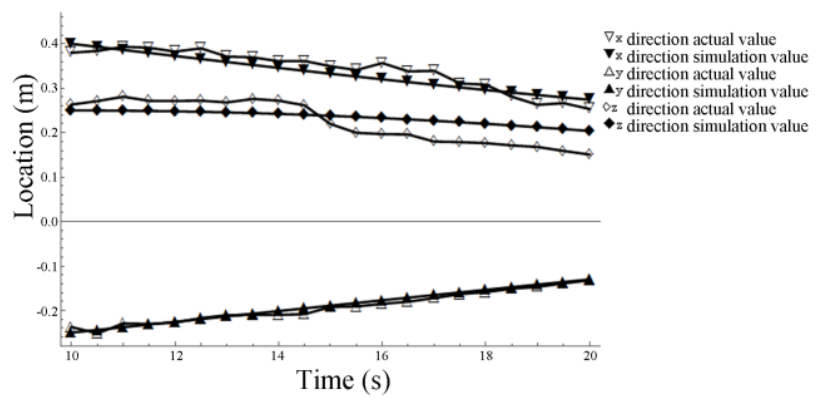

Fig. 14 Hand motion position data and simulation analysis results

Fig. 14 shows that during the joystick pulling process, the coordinate values of the hand decrease along the $\mathrm{X}$ direction, reversely decrease along the $\mathrm{Y}$ direction, and decrease along the $\mathrm{Z}$ direction with rather a large decline in magnitude. The actual values are basically consistent with the simulation values with the same change tendency.

\subsection{Dynamics simulation analysis}

HB6105-86 "Flying Qualities Specification of Military Helicopter" specifies the physiological value of the manipulation force needed to meet the requirements of different flying quality levels (Wang and Zhuang, 2008), as shown in Table 4.

Table 4. Cockpit ultimate manipulation force

\begin{tabular}{cc}
\hline Flying quality level & Ultimate manipulation force/N \\
\hline 1 & 85 \\
2 & 100 \\
3 & 180 \\
\hline
\end{tabular}

Suppose that the pulling force applied to the joystick is $50 \mathrm{~N}$. Because the motion law and load of the hand at the end of the upper limb kinematic chain are already known, the resultant torque of the upper limb joint is derived via the 
inverse kinematics. According to Wang et al (2013), the stress on the shoulder joint is the largest, followed by that on the elbow joint, with the stress on the wrist joint being the smallest. The stress on the shoulder joint is another main factor causing a pilot's arm to experience working fatigue. The driving torque is shown in Fig. 15, in which the torque in the $\mathrm{X}$ direction of the shoulder joint increases negatively with time, while the torque in the $\mathrm{Z}$ direction decreases gradually, with a magnitude of nearly $50 \%$. The torque of the elbow is very small, as is the range of change. The shoulder torque is greater than the elbow torque, and the change tendency based on the simulation experiment is consistent with that reported in the literature (Li et al., 2014), satisfying the law of actual motion. This consistency verifies the correctness of Kane's equation in screw form.

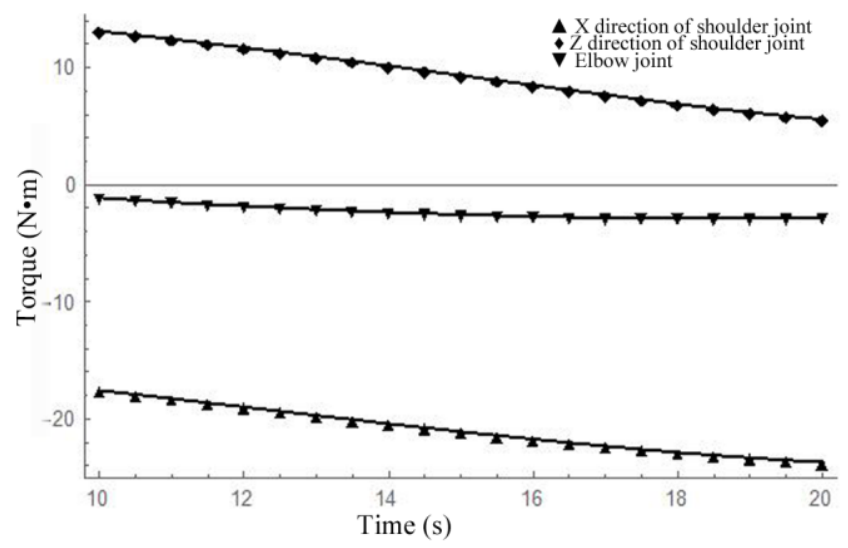

Fig. 15 Driving torque curve

\section{Conclusions}

1) This study establishes a 6-DOF model of upper limbs. Screw theory is used for kinematic analysis, which requires only the establishment of the basic coordinate system and the tool coordinate system to generate a simpler motion model with a more definite physical and geometric significance.

2) Kane's equation based on screw theory is simple in form, with the advantages of both vectorial mechanics and analytical mechanics and none of their shortcomings. Therefore, it is of high computational efficiency and easy to program.

3) In the Mathematica environment, the man-machine system consisting of a pilot and a joystick is taken as the analysis object. A simulation analysis of the process of a pilot in the fiftieth percentile of the Chinese pilot population pushing and pulling a joystick yields the curves of the angular velocity and angular acceleration, indicating that the manipulation comfort is rather good. The correctness of the simulation analysis is verified by comparing the output manipulation process posture data with the simulation data by means of the posture data measurement experiment during the pilot manipulation process. The torque curve shows that the torque of the shoulder joint is greater than that of the elbow joint, and the changing tendency coincides with the actual motion law, thereby verifying the correctness of Kane's equation in screw form. The torque of the joints obtained can be introduced into the comfort evaluation in a follow-up study to improve the manipulation comfort of an aircraft cockpit.

\section{Acknowledgment}

This study was jointly supported by the National Natural Science Foundation of China and Civil Aviation Administration of China (U1333119), the National Defense Basic Scientific Research Project (JCKY2013605B002), the Zhejiang Provincial Department of Education Research Project (Y201636110), and the Major Scientific and Technological Fund in Zhejiang Province (2016C02G2100540)

\section{Conflict of interest}


None declared.

\section{References}

Brockett, R., Robotic manipulators and the product of exponentials formula, Proceedings of the MTNS83 International Symposium (1984), pp. 120-129, Springer, Beer Sheva.

Cao, B. and Yang, J. T., Analysis of the pilot flight fatigue, Chinese Journal of Convalescent Medicine, Vol. 19, Issue 10 (2010), pp. 920-922.

Chen, Q. C., Zhu, S. Q., Wang, X. Y. and Zhang, X. Q., Inverse kinematics sub-problem solution algorithm for serial robot based on screw theory, Journal of Zhejiang University, Vol. 1 (2014), pp. 1-8.

Chen, Z. A., Zhang, M., Qian, P. L. and Yu, T., Research on steering discomfort based on drivers' joint torque, Agricultural Equipment \& Vehicle Engineering, Vol. 54, Issue 7 (2016), pp. 21-29.

Deng, L., Chen, B. and Yu, S. H., Research on evaluation method of upper limb operating comfort for cabin layout optimization, Mechanical Science and Technology for Aerospace Engineering, Vol. 36, Issue 1 (2017), pp. 108-113.

FAA, The national plan for aviation human factors [EB/OL] (1990), Washing DC (accessed, 2013-01-01, http:[C]//www.hf.faa.gov/docs/nat_plan.pdf).

Foyle, D, C. and Hooey, B. L., Human performance modeling in aviation (2008), pp. 20-21, CRC Press, Taylor \& Francis Group, Florida.

GJB 4856-2003, Human dimensions of Chinese Male Pilot Population (2003).

Jung, E. S., Kee, D. Y. and Chung, M. K., Upper body reach posture prediction for ergonomic evaluation models, International Journal of Industrial Ergonomics, Vol. 16, Issue 2 (1995), pp. 95-107.

Jung, K. H., Park, J. W. and Lee, W. S., Development of quantitative ergonomic assessment method for helicopter cockpit design in a digital environment, Journal of the Ergonomics Society of Korea, Vol. 29, Issue 2 (2010), pp. 203-210.

Kong, Y. L., Research on aircraft cockpit comfort evaluation method (2017), Nanjing University of Aeronautics and Astronautics, Nanjing.

Lee, W., Jung, K., Jeong, J., Park, J., Cho, J., Kim, H., Park, S. and You, H., An anthropometric analysis of Korean male helicopter pilots for helicopter cockpit design, Ergonomics, Vol. 56, Issue 5 (2013), pp. 879-887.

Li, H. R., Jiang, Z. B. and Wu, W., Kane dynamic equations based on screw theory for upper limb rehabilitation robot. Journal of Northeastern University (Natural Science), Vol. 35, Issue 2 (2014), pp. 153-157.

Liu, L., Research of aircraft cockpit interior layout design and ergonomics method (2016), Nanjing University of Aeronautics and Astronautics, Nanjing.

Lu, S., Gu, Z.Q., Wu, W. G., Liang, X. B. and Peng, G. P., Comfort analysis of dump truck joystick based on ergonomics, Journal of Central South University, Vol. 44, Issue 9 (2013), pp. 3665-3669.

Ma, L., Zhang, W., Chablat, D., Bennis, F. and Guillaume, F., Multi-objective optimization method for posture prediction and analysis with consideration of fatigue effect and its application case, Computers and Industrial Engineering, Vol. 57, Issue 4 (2009), pp. 1235-1246.

Ma, Z., Xue, H. J. and Su, R. E., Human-modeling and analysis of ergonomics based on Jack, Aeronautical Computing Technology, Vol. 38, Issue 1 (2008), pp. 97-100.

Marler, R. T., Arora, J. S., Yang, J. Z., Kim, H. J. and Abdel-Malek, K., Use of multi-objective optimization for digital human posture prediction, Engineering Optimization, Vol. 41, Issue 10 (2009), pp. 925-943.

Murray, R. M., Li, Z. X. and Sastry, S., A Mathematical Introduction to Robotic Manipulation (1998), China Machine Press, Beijing.

Rocha, C., Tonetto, C. and Dias, A., A comparion between the Denavit-Hartenberg and the screw-based methods used in kinematic modeling of robot manipulators, Robotics and Computer-Intergrated Manufacturing, Vol. 27, Issue 4 (2011), pp. 723-728.

Sariyildiz, E., Cakiray, E. and Temeltas, H., A comparative study of three inverse kinematics methods of srrial industrial robot manipulators in the screw theory framework. International Journal of Advanced Robotic Systems, Vol. 8, Issue 5 (2011), pp. 9-24.

Selig, J. M., Geometric fundamentals of robotics (2008), translated by Yang Xiangdong, Tsinghua University press, 


\section{Beijing.}

Wang, D. Y., Shi, J., Tang, G., Sun, F. and Xu, Z., Simulation and analysis for action of steering lever by pilots, Journal of Donghua University, Vol. 39, Issue 4 (2013), pp. 436-439.

Wang, R. and Zhuang, D. M., Layout optimization of cockpit based on human comfort, Acta Armamentarii, Vol. 29, Issue 9 (2008), pp. 1149-1152.

Xue, H. J., Zheng, S., Tie, Y. Q. and Liu, X., Simulation for pointing manipulation performance of pilot, Journal of Beijing University of Aeronautics and Astronautics, Vol 39, Issue 12 (2013), pp. 1584-1590.

Yang, J. C., Guo, D. and Shi, Y., Comfort modeling and simulation of helicopter cockpit steering apparatus, Computer Engineering and Applications, Vol. 48, Issue 27 (2012), pp. 244-248.

Zhao, D., Lin, Y., Shou, G., Chen, B., Gu, Y. and Wang P., Dynamic analysis and simulation for an active catheter, Journal of Advanced Mechanical Design Systems \& Manufacturing, Vol. 10, Issue 2 (2016), JAMDSM0018-JAMDSM0018. 